\title{
Evolución de los dispositivos electrónicos en un automóvil
}

\section{Evolution of electronic devices in an automobile}

\author{
EdD. Pérez Darquea Diego Gustavo \\ Universidad Internacional del Ecuador, Ecuador
}

Autor para correspondencia: dperez@uide.edu.ec

Fecha de recepción: 14 de Junio de 2017 - Fecha de aceptación: 15 de Noviembre de 2017

Resumen: Este artículo presenta un estudio de la naturaleza y desarrollo tecnológico en la parte electrónica de un automóvil, siendo importante un análisis de las características técnicas, económicas y ambientales a considerar para fabricar una pieza y/o componente de un vehículo, que cumplan con las especificaciones. En este estudio, se discuten las transformaciones profundas ocurridas en los últimos años en la parte eléctrica-electrónica de un vehículo y presenta las ventajas que estos cambios han proporcionado. Los dispositivos electrónicos han evolucionado fundamentalmente en lo relacionado a que sus características permiten responder a las nuevas exigencias reglamentarias y comerciales a las que debe hacer frente el automóvil actual. Los vehículos con el pasar de los tiempos tienen nuevas funcionalidades, empezando por la sustitución de funciones, gestión de elementos a través de software y la diagnosis de fallos y averías. El desarrollo de nuevos sistemas de un vehículo se centra en el diseño de las diferentes configuraciones y elementos electrónicos que controlan el estado del vehículo y como se aplican las diferentes características de comunicación. Las aplicaciones de estos dispositivos electrónicos se han ido implementando paulatinamente en los diferentes sistemas de un vehículo como motor, frenos, transmisión, dirección, seguridad, confort. En definitiva, han venido a reemplazar a los elementos mecánicos y sus funciones.

Palabras Clave: desarrollo tecnológico; automóvil; especificaciones técnicas; dispositivos electrónicos; exigencias reglamentarias

Abstract: This paper review presents a study of the nature and technological development in the electronic part of a car, being important an analysis of the technical, economic and environmental characteristics to be considered in order to manufacture a part and / or component of a vehicle specifications. In this study, we discuss the deep transformations that have occurred in recent years in the electrical-electronic part of a vehicle and present the advantages that these changes have provided. The electronic devices have evolved fundamentally in that their characteristics allow to respond to the new regulatory and commercial requirements to which the present automobile must face. The vehicles with the passing of time have new functionalities, starting with the replacement of functions, management of elements through software and the diagnosis of faults and faults. The development of new systems of a vehicle focuses on the design of the different configurations and electronic elements that control the state of the vehicle and how different communication characteristics are applied. The applications of these electronic devices have been gradually implemented in the different systems of a vehicle such as engine, brakes, transmission, steering, safety, comfort. In short, they have come to replace the mechanical elements and their functions.

Keywords: technological development; automobile; technical specifications; electronic devices; regulatory requirements 


\section{Introducción}

La evolución que está experimentando el sector del automóvil está relacionado con el desarrollo e implementación de dispositivos electrónicos. Por ejemplo, en lo relacionado con la confortabilidad del usuario ha aumentado durante los últimos años debido a la instalación de sistemas como: "control de velocidad de crucero", "sistemas de aire acondicionado", "retrovisores autoregulables", etc. (Lucas Violero, 2016)

En base a las necesidades y especificaciones técnicas de la aplicación automotriz a fabricar, en este caso un dispositivo electrónico, se debe analizar las condiciones de funcionamiento de la pieza, de manera individual y en conjunto; tomando en cuenta aspectos técnicos de diseño y fabricación. Como todo elemento de uso automotriz debe pasar por rigurosos métodos de evaluación y pruebas, para cumplir con las especificaciones (Parera, 2000).

El fabricante en función de las condicionantes de cada momento diseña y fabrica un vehículo que solvente los requisitos sociales y legales utilizando las tecnologías disponibles, que den como resultado un mejor producto al costo más apropiado (María Fernández-Albalat, 2017).

La demanda de prestaciones de la sociedad ha sufrido grandes cambios. En los años 50, cuando los automóviles comenzaban a popularizarse, tener un automóvil era considerado suficiente, y la sociedad no demandaba muchas prestaciones.

En los años 60 y años 70 se dio un aumento de las prestaciones de los vehículos, ya que la sociedad respondía favorablemente a la evolución tecnológica hacia automóviles más grandes y más veloces a precios más económicos, desarrollo tecnológico de la parte electrónica y, mecánica de los sistemas del vehículo.

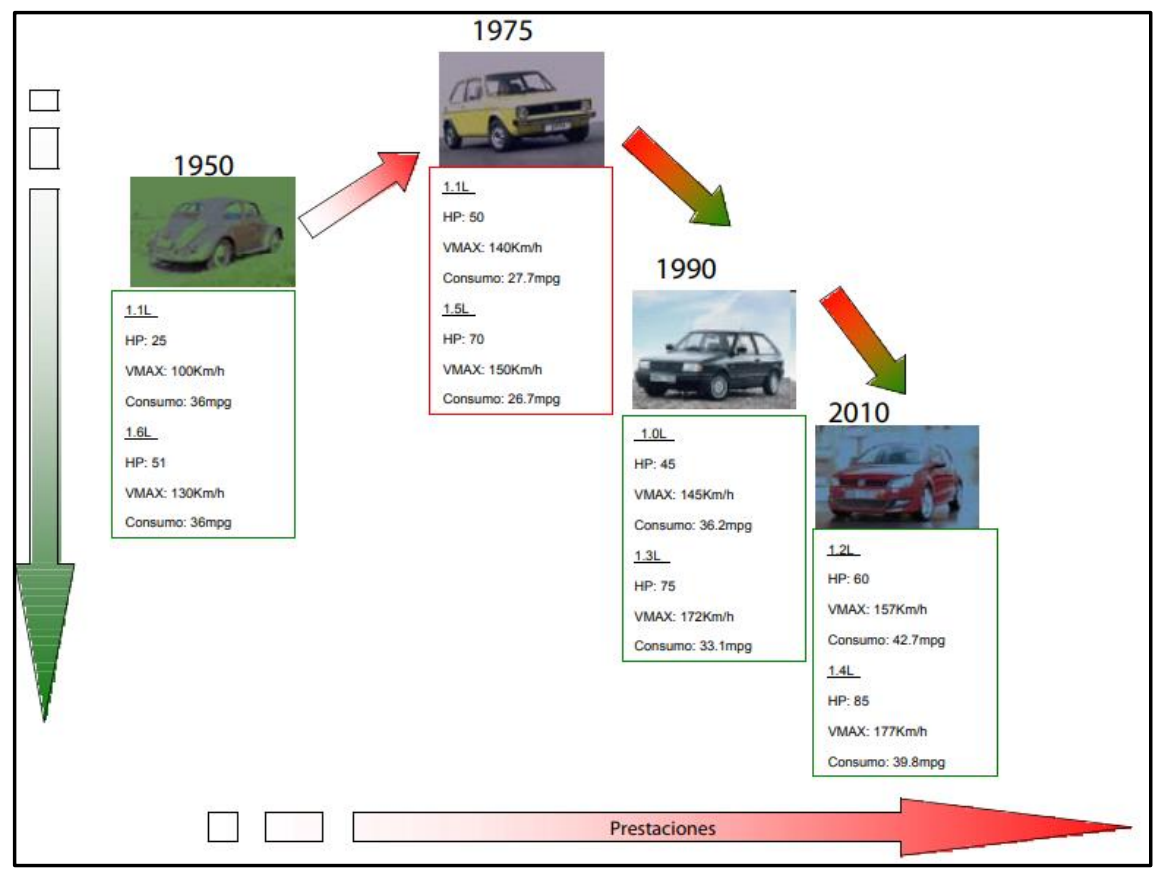

Figura 1. Evolución en las prestaciones de un vehículo Fuente: Ministerio de Energía, Turismo y Agenda Digital, 2018 


\section{Materiales y Métodos}

En la electrónica del automóvil, se ha obtenido desarrollos en los siguientes dominios (Cook, 2006):

1. Grupo motopropulsor

$\checkmark$ Gestión electrónica del motor con regulación de inyección, encendido, y anticontaminación.

$\checkmark$ Gestión electrónica de la caja de velocidades (automática, manual, por correa).

$\checkmark$ Gestión electrónica de la refrigeración motor.

$\checkmark$ Gestión electrónica de la sobrealimentación.

$\checkmark$ Motorización híbrida.

2. Seguridad

$\checkmark$ Sistemas de antibloqueo de los frenos.

$\checkmark$ Control dinámico de la estabilidad.

$\checkmark$ Suspensión activa.

$\checkmark$ Dirección asistida.

$\checkmark$ Iluminación de xenón.

$\checkmark$ Ilumianción dinámica.

$\checkmark$ Indicador de mantenimiento.

$\checkmark$ Control la presión de los neumáticos.

$\checkmark$ Sistemas de retención suplementaria (Airbags y pretensores).

\section{Confort}

$\checkmark$ Regulación adaptativa de la velocidad.

$\checkmark$ Control de acceso sin llave.

$\checkmark$ Cierre centralizado.

$\checkmark$ Climatización automática.

$\checkmark$ Memorización del puesto de conducción.

$\checkmark$ Automatismo de limpieza e iluminación.

$\checkmark$ Ayuda de aparcamiento.

4. Comunicación

$\checkmark$ Audio.

$\checkmark$ Vídeo.

$\checkmark$ Ordenador de a bordo.

$\checkmark$ Mandos vocales.

$\checkmark$ Telefonía móvil.

$\checkmark$ Navegación.

$\checkmark$ Pantalla centralizada.

$\checkmark$ Visualización en el parabrisas.

\section{Orígenes y evolución}

En los años 60, se inició con la primera etapa, en la cual se realizaban trabajos de sustitución de partes mecánicas por otras electrónicas con el objetivo de mejorar la fiabilidad. A 
manera de ejemplo tenemos, en 1965, el encendido por ruptor dio paso al encendido transistorizado. Con lo cual, se suprimían los efectos negativos de la erosión de los contactos del ruptor de encendido.

Nishiguchi hace un análisis actual e histórico de la fabricación de vehículos enfocados en la parte mecánica y electrónica, tanto en Japón como Estados Unidos de América, donde se observa que hasta la década de 1960 un vehículo convencional estaba básicamente conformado por componentes mecánicos; la integración de componentes electrónicos inicia su ascenso en los años setenta. Si se compara un vehículo típico de los años sesenta con los vehículos actuales, las diferencias cuantitativas son significativas, pero las transformaciones cualitativas son más profundas (Nishiguchi, 1994).

Posteriormente, aparecieron nuevos sistemas que no podrían haberse desarrollado sin contar con la electrónica. En 1978 llegó el ABS. Por razones de coste, los sistemas electrónicos al principio solo formaban parte de los vehículos de alta gama. A partir de ahí, el sector del automóvil no cesó de ser testigo de continuas innovaciones de módulos y unidades electrónicas.

El despegue de la electrónica digital, a base de transistores, marca verdaderamente la era de la electrónica del automóvil debido a la posibilidad de disminuir considerablemente el tamaño de los componentes. Al hacer componentes electrónicos más pequeños (microscópicos) ha permitido la integración de un mayor número de funciones en el interior de un mismo calculador o centralita (Lara Rivero, (2014).). En lo referente a los cambios ocurridos en los componentes y los sistemas electrónicos han transformado la naturaleza de los vehículos automotores. Se conoce que el $90 \%$ de la innovación que se ha producido en la industria automotriz está relacionada con los componentes electrónicos, y de los cuales el $80 \%$ se relaciona con el software (Hardung, (2004, September)).

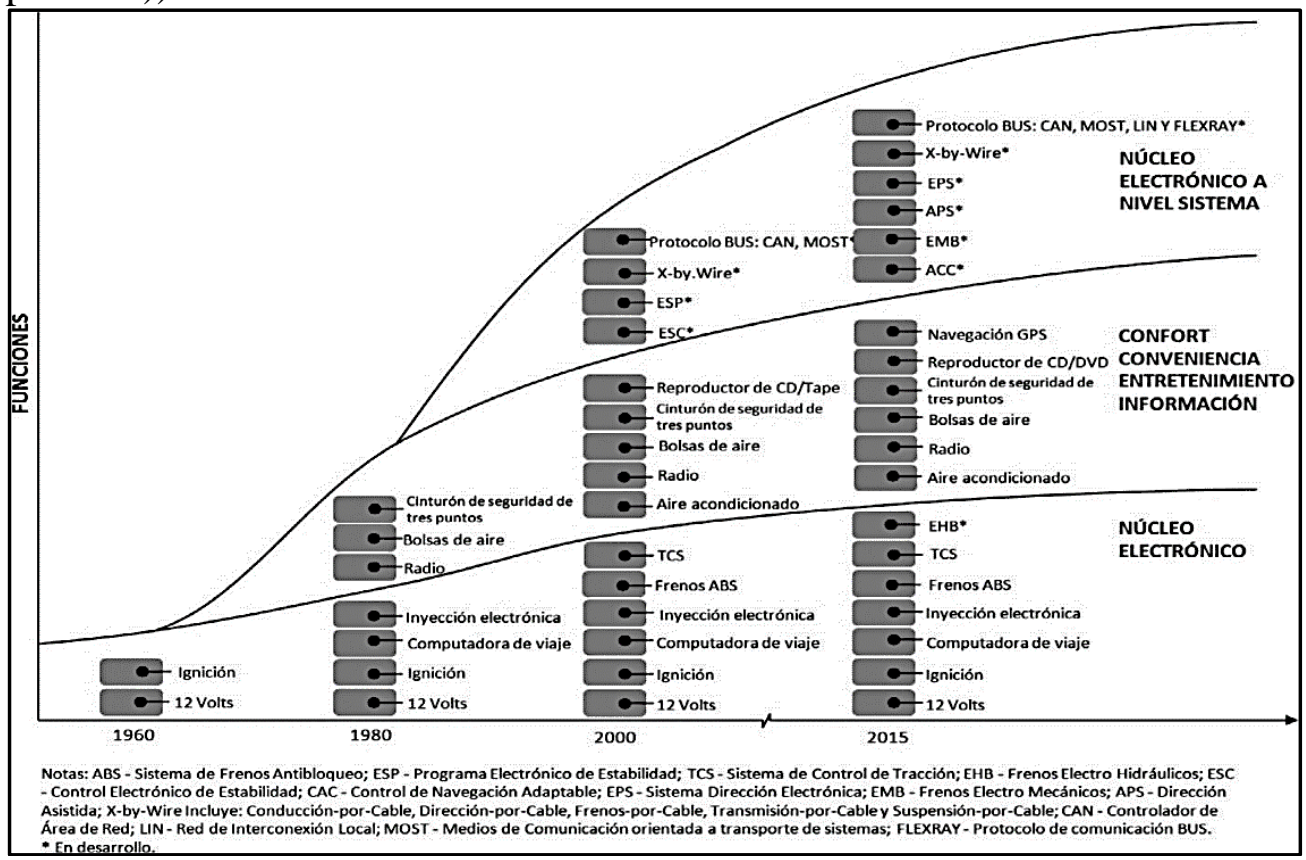

Figura 2: Innovación y evolución de las funciones electrónicas en un automóvil. Fuente: (Lara, 2014). 
Existen también trabajos relacionados con la parte de la reducción en la siniestralidad que ha experimentado el automóvil en su historia. Gracias a ellos, conducir hoy es casi 200 veces menos peligroso que hacerlo en 1960.

Los microprocesadores o unidades de control electrónico (ECU, por sus siglas en inglés) tuvieron su desarrollo a principios de la década de 1970, se introdujo el sistema electrónico de inyección de combustible; aire acondicionado automático (1971), sistema electrónico de control de frenos (1971), sistema de encendido electrónico (1973), etc. (Abernathy, 1978)

Asimismo, en la seguridad activa se hace referencia a la aplicación de la microelectrónica a los sistemas de control tradicionales. A manera de ejemplo podemos citar, Los ESP Elektronisches Stabilitätsprogramm (en alemán "Programa Electrónico de Estabilidad", abreviado ESP) lograron quitar por completo el problema de la pérdida de control, garantizando que un conductor pueda tener seguridad en el manejo durante el uso del vehículo (MARTIN, 2014).

La electrónica toma una gran importancia en el desarrollo del automóvil debido al enfoque en materiales ligeros, miniaturización, inteligencia, movilidad, energía y sustentabilidad. La evolución de la electrónica de consumo ha dado lugar a requisitos más exigentes para las comunicaciones y las funciones de entretenimiento de los vehículos. Por lo que el desarrollo de control de seguridad y comunicaciones y electrónica de entretenimiento han tenido un rápido crecimiento (París, (2003).).

En vista de esta evolución las principales empresas manufactureras de autos considerarán a la electrónica de automóviles como el factor clave para competir por un mercado de clientes. La innovación se está volviendo cada vez más interdisciplinaria, involucrando industrias como las ciencias de los materiales (materiales ligeros), la industria química (baterías) y la electrónica (comunicaciones y sistemas de entretenimiento) http://saberesyciencias.com.mx/2017/06/04/laimportancia-de-la-electronica-en-el-desarrollo-del-automovil/.

Existe también una evolución en cuanto a la transmisión de datos. En la primera evolución de los arneses la principal y exclusiva función de los cables era conducir electricidad. En la segunda se produce cuando los cables conducen electricidad e información; este sistema se caracteriza por un cable/una señal. Y la tercera se caracteriza porque el cable puede transmitir más de una señal (sistemas multiplexos) (Figueroa Peñafiel, 2015).

En los sistemas automotrices se sustituyen los sistemas mecánicos a electrónicos (ver figura 3). Por ejemplo, la unidad de control electrónico es el corazón de un vehículo debido a las funciones que debe cumplir control de la combustión; hay un gran número de computadoras montadas sobre el vehículo que controlan diferentes sistemas. Hoy en día un vehículo típicamente contiene alrededor de 25 a 35 microcontroladores, y los de lujo llegan a tener aproximadamente de 60 a 150. 


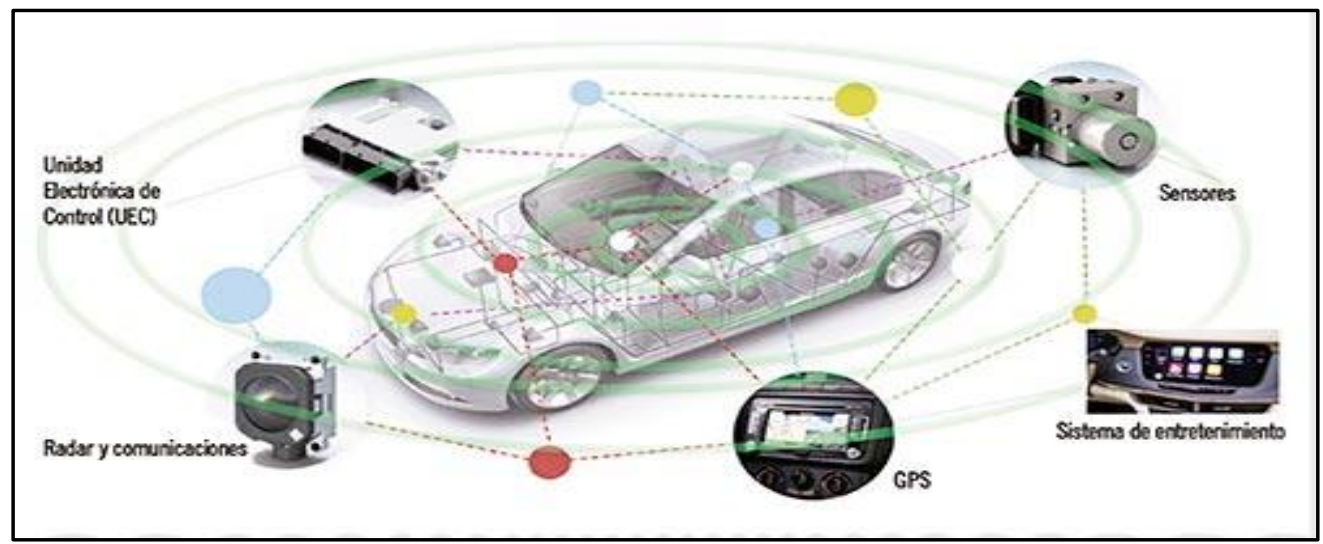

Figura 2: Sustitución de los sistemas mecánicos a los sistemas electrónicos. Fuente: Ambrosio Lázaro \& Sánchez Gaspariano, 2004

Todos estos avances han conducido a un ritmo acelerado de innovación del diseño y manufactura de los ECU, sensores, solenoides y arneses, así como a la administración del volumen de energía e información asociados a una red cada vez más extensa e interdependiente (Bonet, 2017).

\section{Conclusiones}

Los vehículos responden a una serie de condicionantes y estas proporcionan las claves de las características de los vehículos del futuro.

Los involucrados en la industria automovilística se enfrentarán a mayores problemas en los próximos años, ya que deben mantener el equilibrio entre las presiones del consumidor, los requerimientos de seguridad y los factores económicos.

Las empresas están construyendo estrategias de solución que les permitan enfrentar la creciente complejidad, disminuir el número y la diversidad de desequilibrios tecnológicos, así como mejorar los procesos de innovación.

Los sistemas electrónicos actuales tienen que poseer unas prestaciones insustituibles. En la evolución de la red de componentes electrónicos, es muy importante tener en cuenta la innovación del resto de sistemas que conforman un automóvil.

\section{Bibliografía}

Abernathy, W. J. (1978). Patterns of industrial innovation. Technology review.

Ambrosio Lázaro, R. \& Sánchez Gaspariano, L. A. (2004). La importancia de la electrónica en el desarrollo del automóvil. Rettrive from http://saberesyciencias.com.mx/2017/06/04/laimportancia-de-la-electronica-en-el-desarrollo-del-automovil

Bonet, E. V. (2017). La revolución electrónica (Vol. 8). Ed. Universidad de Cantabria.

Cook, J. A. (2006). Cook, J. A., Sun, J., Buckland, J. H., Kolmanovsky, I. V., PenAutomotive 
powertrain control—a survey. Asian Journal of Control. 8(3), 237-260.

Figueroa Peñafiel, H. J. (2015). Estudio y análisis del sistema multiplexado del vehículo híbrido toyota prius.

Hardung, B. K. ((2004, September)). Reuse of software in distributed embedded automotive systems. In Proceedings of the 4th ACM international conference on embedded software. ACM, pp. 203-210.

Lara Rivero, A. Á. ((2014).). De sistema mecánico a sistema tecnológico complejo: El caso de los automóviles. . En Contaduría y administración. (págs. 59(2), 11-39.).

Lucas Violero, F. J. (2016). Software de simulación del comportamiento de sistemas de control de vehículos en función de parámetros electrónicos y medioambientales.

María Fernández-Albalat, R. D. (2017). Estudio de mercado de la incorporación de sistemas de confort, asistencia y seguridad en los vehículos.

MARTIN, A. G. (2014). Actualización e implementación del sistema de configuración y chequeo eléctrico-electrónico de vehículos en Ford Valencia (Doctoral dissertation).

Ministerio de Energía, Turismo y Agenda Digital (2018). Retrive from http://www.minetad.gob.es/industria/observatorios/sectorautomocion/actividades.pdf. (2010).

Nishiguchi, T. (1994). Strategic industrial sourcing: The Japanese advantage. Oxford University Press on Demand.

Parera, A. M. (2000). Sistemas de seguridad y confort en vehículos automóviles. Marcombo.

París, A. P. ((2003).). Aplicaciones electronicas del automovil 2. Vivat Academia, (25), 37-48. 